\title{
BMJ Open Measuring functional ability in healthy ageing: a nationwide cross-sectional survey in the Philippine older population
}

\author{
Dario Moreno-Agostino (D) , ${ }^{1,2,3}$ Matthew Prina, ${ }^{1}$ Kia-Chong Chua, ${ }^{1}$ \\ Amuthavalli Thiyagarajan Jotheeswaran, ${ }^{4}$ Ritu Sadana, ${ }^{4}$ Alana Officer, ${ }^{5}$ \\ Kaloyan Kamenov (D) , ${ }^{6}$ Alarcos Cieza ${ }^{6}$
}

To cite: Moreno-Agostino D, Prina M, Chua K-C, et al. Measuring functional ability in healthy ageing: $\mathrm{a}$ nationwide cross-sectional survey in the Philippine older population. BMJ Open 2021;11:e050827. doi:10.1136/ bmjopen-2021-050827

- Prepublication history and additional supplemental material for this paper are available online. To view these files, please visit the journal online (http://dx.doi.org/10.1136/ bmjopen-2021-050827).

Received 04 March 2021 Accepted 03 September 2021

Check for updates

(C) Author(s) (or their employer(s)) 2021. Re-use permitted under CC BY-NC. No commercial re-use. See rights and permissions. Published by BMJ.

For numbered affiliations see end of article.

Correspondence to Dr Dario Moreno-Agostino; d.moreno@ucl.ac.uk

\section{ABSTRACT}

Objectives To analyse the empirical support of the functional ability concept in the healthy ageing framework developed by the WHO in a sample of the Philippine older population. According to this framework, environmental factors may enhance or hinder functional ability, which is the person's ability to do what they value, broadly represented by subjective well-being. Moreover, this network of relationships may be moderated by personal characteristics such as gender.

Design Cross-sectional observational study.

Setting Philippines, general population.

Participants Respondents of the 2016 National Disability Prevalence Survey/Model Functioning Survey aged 50+ $(\mathrm{N}=2825)$.

Primary and secondary outcome measures Latent (unobserved) measures of functional ability, environmental factors (physical environmental factors and social network and support) and subjective well-being (positive affect, negative affect and evaluative well-being) were obtained from different items from the survey questionnaire using a SEM framework.

Results We found that the relationship between environmental factors and the three components of subjective well-being considered in this study was partially explained by differences in functional ability. The portion of those effects accounted for by functional ability was comparatively larger for the physical than for the social environmental factors. We found no evidences of gender differences in this network of relationships.

Conclusions These findings suggest the relevance of functional ability at explaining the relationship between environmental factors and subjective well-being in older adults. Future studies may replicate these findings longitudinally and including other relevant measures as the person's objective level of intrinsic capacity.

\section{INTRODUCTION}

Healthy ageing has been defined by the $\mathrm{WHO}$ as the process of developing and maintaining the functional ability that enables well-being in older age. ${ }^{1}$ The key term in this definition is functional ability, which refers to
Strengths and limitations of this study

- We used a structural equation modelling approach to empirically test the concept of functional ability from a healthy ageing perspective.

- We tested the potential impact of different operationalisations of functional ability in the networks of relationships tested, as well as potential gender differences in these networks.

- We used a multidimensional approach to subjective well-being in older adults.

- We could not include objective intrinsic capacity measures in the analyses due to their absence from the questionnaires.

- All data analysed were cross-sectional, so there is room for reverse causation.

health-related attributes that enable people to be and do what they have reason to value. Three components determine functional ability: (1) intrinsic capacity, which is the composite of all mental and physical capacities that people can draw on at any point in time; (2) environmental factors, including those of the home, community and broader society, which can act as barriers or facilitators; (3) the interplay between intrinsic capacity and environmental factors which in other contexts is also called functioning or performance. Therefore, although intrinsic capacity is the main responsible factor for defining what the person might be able to do, the degree to which the environmental characteristics act as barriers or facilitators is what in the end determines what the person is really able to do with those capacities, thus increasing or decreasing their functional ability. ${ }^{2}$

This framework prevents putting the attention exclusively on the person's capacities and highlights the importance of environmental 
characteristics as crucial determinants. If declines in mental and physical capacities are offset by supportive environments, the implications for both older people and society in general are likely to be much more positive than without support. Similarly, non-supportive or harmful environmental characteristics can limit what the person may be able to do, thus reducing their functional ability under their actual intrinsic capacity levels. These definitions of healthy ageing are endorsed by the WHO global strategy and action plan, ${ }^{3}$ which set out an agenda and action plan to maximise functional ability within populations. Rather than solely focusing on preventing or treating diseases in older age, a focus on healthy ageing has the potential to inform public policy, clinical practice and research, with evidence supporting improved outcomes when using holistic approaches in older age, rather than disease-based models of care. ${ }^{4}$

Although people value different things over the life course, some of the experiences that have been acknowledged as important in older age include having a role or identity, having relationships, the possibility of enjoyment, autonomy, security and the potential for personal growth. In the WHO report on ageing and health, ${ }^{1}$ five domains have been mapped to allow people to achieve these ends and to enable older people to do the things that they value, leading to subjective well-being. As further described in WHO's most recent baseline report for the decade of healthy ageing, ${ }^{5}$ functional ability comprises diverse relevant aspects that draw on physical, cognitive and sensory capacities, to enable people to meet their basic needs; learn, grow and make decisions; be mobile; build and maintain relationships; and contribute.

Evidence on the operationalisation and predictive validity of intrinsic capacity, a component of functional ability, has started to emerge. ${ }^{46}$ Beard and colleagues, using information on biomarkers and self-reported measures, identified one general factor and five subfactors of intrinsic capacity that included locomotor, cognitive, psychological, sensory and vitality in the English Longitudinal Study of Ageing. Recent evidence has supported the generalisability of these findings, showing the adequacy of this same factor structure in a cohort of Chinese older adults. ${ }^{7}$ Similarly, using the same intrinsic capacity operationalisation, recent studies have shown that intrinsic capacity is associated with a reduced ability to meet the basic needs and to be mobile, along with other adverse outcomes in Chinese ${ }^{8}$ and Mexican ${ }^{9}$ older populations. However, similar evidence on functional ability is lacking. Therefore, we decided to assess whether the theoretical framework of functional ability can be supported by empirical data, using the Model Disability Survey (MDS) from the Philippines. The Philippine MDS dataset encompasses a very comprehensive set of measures of functional ability, environmental factors and subjective well-being. Although this dataset lacks objective measures of intrinsic capacity, the detailed information on the other components provides the opportunity to comprehensively measure functional ability and test the existing network of relationships between this and other constructs present in the healthy ageing framework, that is, environmental factors and subjective well-being. Additionally, the Philippine MDS dataset also provides the opportunity to explore the appropriateness of this framework in a low- and middle-income country.

We aimed to investigate the empirical support for the concept of functional ability within the context of healthy ageing, considering the relative absence of empirical evidence on its utilisation. Thus, we analysed whether more supportive environmental characteristics are related to better subjective well-being directly (ie, direct effects) or if, in line with the WHO framework, this relationship can be explained by an increase in the person's level of functional ability that, in turn, leads to a higher subjective well-being (ie, indirect effects). Moreover, this network of relationships is expected to be modified by personal characteristics such as gender. ${ }^{1}$ Thus, we also analysed the moderating role of gender as a way to analyse potential gender inequalities in healthy ageing.

\section{METHODS}

\section{Study Sample}

Data from the nationally representative cross-sectional 2016 National Disability Prevalence Survey, also called the Model Functioning Survey (NDPS/MFS 2016), were analysed. This survey used the MDS questionnaire developed by WHO and the World Bank, including questions on the sociodemographic characteristics, environmental factors, functioning and well-being, among other aspects. The questionnaire and its manual are publicly available in the WHO webpage. ${ }^{10}$ The survey was administered via face-to-face interviews at the participants' households by trained interviewers between 21 November and 13 December 2016. A two-stage stratified sample design based on the Census of Population and Housing was used to obtain a representative sample of the population aged 15 years or older. Further details on the survey methodology used in the NDPS/MFS 2016 can be found in the Philippine Statistics Authority webpage, ${ }^{11}$ whereas the full questionnaire can be accessed from the International Household Survey Network. ${ }^{12}$ Due to this study's focus on the older population, we used the data corresponding to those participants aged $50+$ that completed the interview and provided informed consent. Thus, the sample for the present study comprised 2825 participants.

The MDS was developed by WHO and the World Bank with the active involvement of the public, including people with disability and their representative organisations, academic and research organisations, disability and development organisations, national statistical offices, health and social development ministries and UN agencies whose mandate includes disability data. A preliminary draft of the survey was reviewed by representatives of different organisations at a meeting held in December 2012 at the WHO Headquarters in Geneva. Public was also involved during the cognitive testing and piloting phase 
to ensure that MDS questionnaire was valid and generates reliable estimates. Three rounds of cognitive testing of the MDS were carried out in 2013, 2014 and 2015 in the USA, Norway, Malawi, Cambodia, China, Nepal and the UK. Reports from these tests were reviewed and discussions were held by $\mathrm{WHO}$ and experts from the public to inform revisions to the MDS instrument. A similar approach was applied during the pilot testing which took place in Cambodia, Malawi, Pakistan and Oman.

\section{Patient and public involvement}

Patients and the public were not involved in the planning and design of this analysis but were involved in the development of MDS.

\section{MEASURES}

\section{Functional ability}

Information on functional ability was drawn from the Module 4000 of the survey questionnaire. This module is grounded on the International Classification of Functioning by the $\mathrm{WHO},{ }^{13}$ including 48 questions on the problems experienced in a range of domains of activities and participation (eg, mobility, hand and arm use, selfcare, sensory functioning, interpersonal relationships, cognition). The participant is asked to what extent, in the past 30 days, performing the specific activity or experiencing the corresponding event constituted a problem for them, and given a 5-point response scale from 1 ('not a problem') to 5 ('extreme problem'). Since these problems can be offset (or exacerbated) by environmental characteristics, they reflect the person's level of functional ability rather than their level of intrinsic capacity. Given the focus on older adults and the high proportion of missing data in those items (above $10.0 \%$ and up to $97.7 \%$ ), four questions regarding work and schooling were not considered in the main analyses. Similarly, a question regarding problems with intimate relationships was not considered due to the high proportion of missing data $(13.3 \%)$. Thus, 43 items were considered for the analyses. The full description of these items is available in the Module 4000 of the survey's individual questionnaire. ${ }^{12}$ The list of items in this module, along with the number and percentage of missing cases in the sample, is available in table 1 .

\section{Environmental factors}

Environmental factors were operationalised by means of two main aspects: physical environmental factors and social network and support. Although these two dimensions do not exhaustively cover 'all factors in the extrinsic world that form the context of an individual's life' as per the WHO framework, ${ }^{1}$ they have been highlighted in previous literature as relevant dimensions covering relevant aspects of physical and psychosocial environments. ${ }^{14} 15$ The participants' physical environmental factors were assessed with a set of nine questions on the degree to which several infrastructures and environmental conditions (eg, health facilities, means of transportation, dwelling, etc) make it hard or easy for the person to use them. These questions had a 5-point response scale ranging from 1 ('very easy') to 5 ('very hard'). As in the case of functional ability, a question on the workplace or educational institution conditions was excluded due to the high number of missing data $(24.2 \%)$. On the other hand, participants' social network and support were assessed by means of six questions on (1) the degree to which the person can rely on different people to get help from and (2) the degree of closeness of the person's relationship with (i) family members, (ii) friends or coworkers and (iii) neighbours. A question on the closeness of the relationship with the spouse or partner was excluded due to the high number of missing data $(33.5 \%)$. These questions had a 5-point response scale ranging from 1 ('very easy/'very close') to 5 ('very difficult'/'not at all close'). Questions on the physical environmental factors and social network and support were drawn from the sections on 'Hindering or facilitating environment' and 'Support and relationships', respectively, of the Module 3000 of the survey's individual questionnaire. ${ }^{12}$ The list of items in these sections, along with the number and percentage of missing cases in the sample, is available in online supplemental table S1.

\section{Subjective well-being (SWB)}

Given the multidimensional nature of SWB, and the fact that different components of SWB may be differently related to third variables, ${ }^{16}{ }^{17}$ we separately considered measures of positive affect (PA), negative affect (NA) and evaluative well-being. Measures on evaluative wellbeing included a general rating of the overall quality of life in the past 30 days, as well as five questions on the satisfaction with different aspects of life (ie, health, ability to perform daily living activities, 'yourself', personal relationships and the conditions of the living place), which have also been named 'domain satisfaction' in the SWB literature. ${ }^{18}$ The questions had a 5-point ranging from 1 ('very good'/'very satisfied') to 5 ('very bad' /'very dissatisfied'). PA and NA were operationalised by questions on the extent to which the person felt happy, enthusiastic and content (PA), and angry, frustrated, sad, stressed, worried and bored (NA) during the previous day. The response scale of these questions ranged from 1 ('not at all') to 5 ('extremely'). All these items were drawn from Module 7000 of the survey's individual questionnaire. ${ }^{12}$ Questions on the level of energy/tiredness, pain and loneliness, along with a question on whether the person had enough money to meet their needs, were not included in any of the SWB factors due to the consideration that they were indicators of different constructs. The list of items in this module, along with the number and percentage of missing cases in the sample, is available in online supplemental table S2.

\section{Statistical analyses}

In order to explore the relationships between functional ability, environmental factors and SWB in older adults, 
Table 1 Functional ability items included in the models

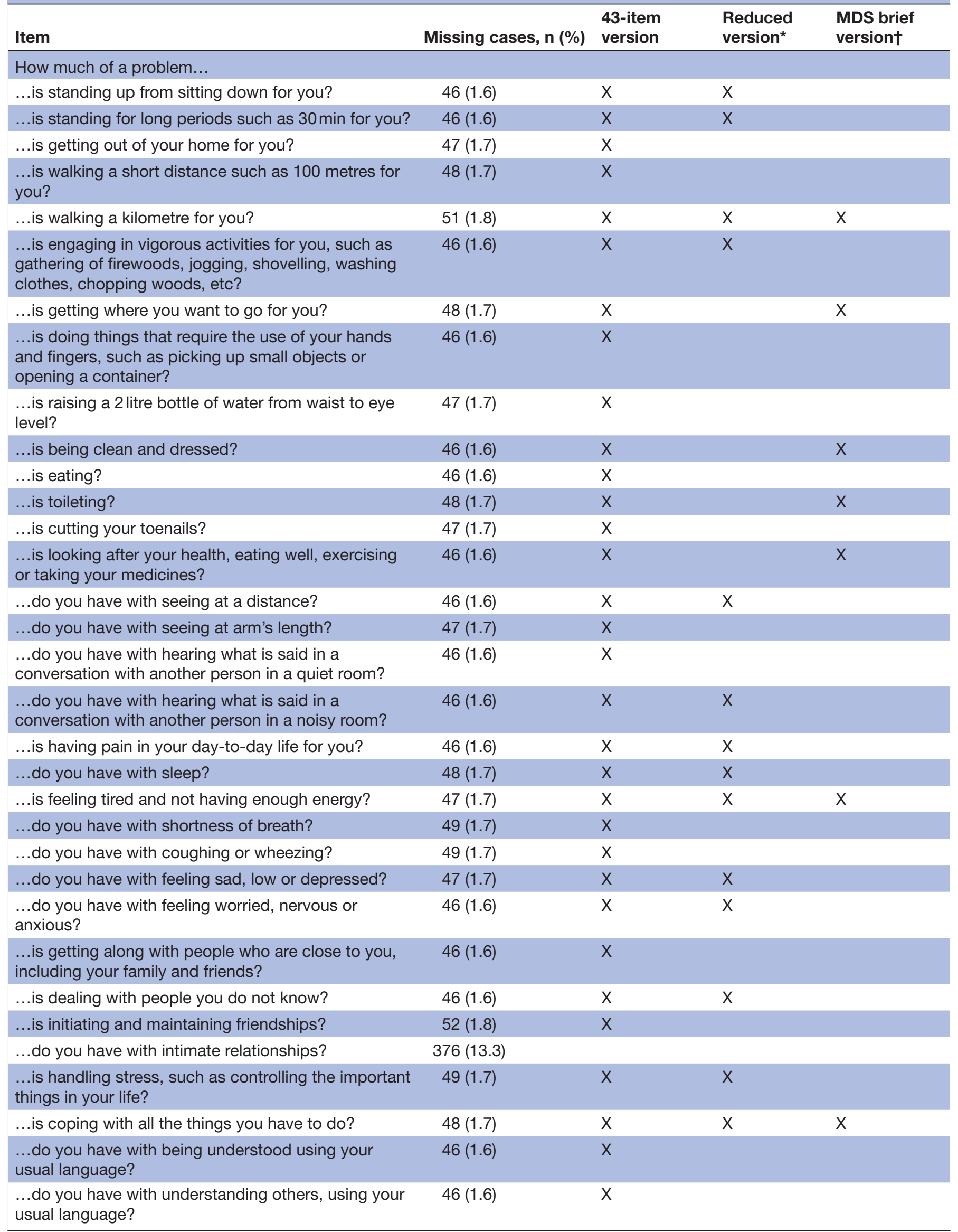




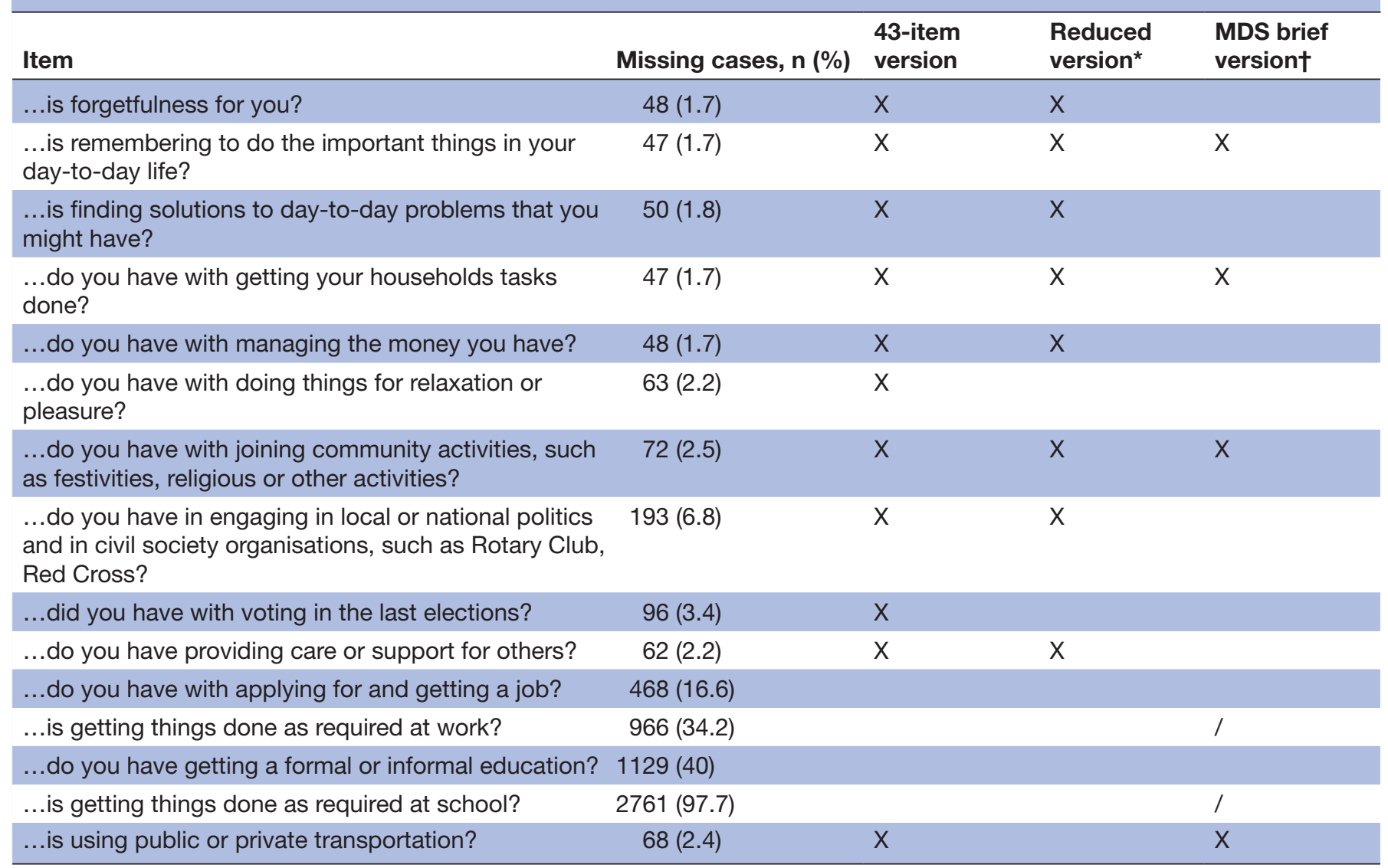

$\mathrm{X}$ indicates item present; / indicates that, in the MDS brief version, a combination of the two questions is used.

*Version used in the main analyses after item selection.

†Brief version proposed by the WHO and the World Bank.

as well as the potential moderating effect of gender in this network, we used a multigroup structural equation modelling (SEM) approach. ${ }^{19}$ First, latent (ie, unobserved) factors were identified for each of the involved variables (physical environmental factors, social network and support, functional ability and SWB). Functional ability, both environmental factors, evaluative well-being and PA items were inversely recoded, so the latent factors reflected higher levels of the corresponding construct. In order to facilitate the estimation of the SEM models, only a subset of the 43 available items on functional ability were used. The item selection was performed based on the amount of variability (and, thus, information) within the items. The five response categories of each item were sorted according to their frequency, and the ratio from the first to the second most populated categories was calculated. Items with a ratio above $70 / 30$ were considered to have a low variability (ie, with the majority of the responses grouped in the most populated category), and therefore were discarded from the models in favour of those items containing a higher amount of information. To examine the potential impact of this empirically driven item selection, factor scores of the reduced functional ability factor were compared with those of the 43-item functional ability factor using a confirmatory factor analysis (CFA) approach.

Once the subset of functional ability items was defined, separate SEM models were built for each of the SWB components considered in the present study (ie, evaluative well-being, PA and NA). According to the WHO healthy ageing framework, more supportive environments may enhance SWB not only directly (ie, direct effects) but also through increased functional ability (ie, indirect effects). To capture these direct and indirect effects, the two environmental latent factors were allowed to correlate with each other and to have direct effects on both functional ability and SWB, whereas functional ability was also hypothesised to have a direct effect on SWB. Age was included as an exogenous variable to help explain individual differences in both functional ability and SWB. No effect was specified from age to any of the environmental factors as, in line with the WHO healthy ageing framework, these factors exist in the 'extrinsic world that form the context of an individual's life ${ }^{11}$ and, therefore, may be best represented as exogenous variables that are not affected by personal characteristics. Model fit was assessed by means of a set of fit indices (ie, $\chi^{2}$, Comparative Fit Index (CFI), Tucker-Lewis Index (TLI) and the 
Root Mean Square Error of Approximation (RMSEA)). CFI and TLI values higher than 0.95 and RMSEA values lower than 0.06 were considered as good fit. ${ }^{20}$

In order to explore to what extent these models varied depending on the gender, the SEM models were built within a multigroup framework. Nested SEM models with increasing constraints were estimated to investigate the level of invariance (ie, equivalence) achieved across genders. Due to the aim of the present study, and the ordinal nature of the indicators, the levels of invariance tested were (1) configural invariance (the same SEM models are specified across groups); (2) strong invariance (loadings and thresholds of the items are constrained to be equal across groups); and (3) regressions invariance (regression coefficients are constrained to be equal across groups). Each level of invariance was considered to be met if the absolute difference in fit compared with the previous model was below 0.01 in both CFI and RMSEA indices, ${ }^{21}{ }^{22}$ provided that the goodness-of-fit was found adequate following the abovementioned guidelines.

Once the level of multigroup invariance was examined for each of the SWB outcomes, the indirect effects (via functional ability) of the two environmental factors on the SWB components were computed as the product of the coefficients of the two paths (environmental factor on functional ability and functional ability on SWB). The total effects of environmental factors on SWB were computed as the sum of the direct and indirect effects. In order to account for the non-normality of the distribution of the indirect and total effects parameters, their statistical significance was tested using resampling methods (ie, non-parametric bootstrap, 1000 bootstrap draws). Due to the lack of a meaningful metric in the latent scores, standardised direct, indirect and total effects were preferred; however, the corresponding unstandardised effects, along with the $95 \%$ CIs for the direct effects, and the $95 \%$ bootstrapped CIs for the indirect and total effects, were reported. Due to the ordinal nature of the items used to identify the latent factors, diagonally weighted least squares estimator was used.

As a sensitivity analysis to investigate the potential impact of using a different functional ability operationalisation, the SEM models were also built using the brief version of the $\mathrm{MDS}^{23}$ to guide the item selection for the functional ability latent variable. This shorter version was not used as the primary approach due to the large proportion of missing data in some of the included items (those related to work and schooling). The level of multigroup invariance achieved, along with the direct and indirect effects in these sensitivity models, was compared with the main models to assess the robustness of the findings.

The analyses were performed using a complete-case approach (ie, including only participants with no missing information on any of the indicators). Such approach assumes that data are missing completely (or unconditionally) at random. As a sensitivity analysis, the results of the main models (models with the highest level of invariance achieved) using the complete-case approach were compared with those of the same models after imputing the missing data. This latter approach relies on the more flexible assumption that data are missing conditionally at random (ie, missingness can be accounted for by the observed data). ${ }^{24}$ Data were imputed using multiple imputation with chained equations (random forest, 20 imputations), including all variables (age, gender, the functional ability, environmental factors and SWB indicators) in the models.

All analyses were performed in R V.3.6.3, ${ }^{25}$ using the lavaan package V.0.6-5 ${ }^{26}$ for the SEM models and the mice package V.3.11. $0^{27}$ for the multiple imputation.

\section{RESULTS}

After excluding participants with missing information in any of the indicators $(n=509)$, the analytic sample comprised 2316 older adults. The analytic sample was younger $(\mathrm{M}=62.01, \mathrm{SD}=9.18)$ than the dropped sample $(\mathrm{M}=65.27, \mathrm{SD}=10.57)$, a difference that was statistically significant $(t(609)=-6.20, \mathrm{p}<0.001)$ and had a lowmedium effect size associated (Cohen's $d=0.35$ ). The analytic sample comprised $54.0 \%$ of women $(n=1256)$, whereas the dropped sample comprised a slightly higher percentage $(59.0 \%, \mathrm{n}=272)$, a difference that was nonsignificant $\left(\chi^{2}(1)=3.00, p=0.08\right)$ and had a small effect size associated (Cramer's $V=0.03$ ).

All the functional ability items included in the MDS, along with the number of missing cases in each of them and the results of the item selection are detailed in table 1. Among the 43 items considered in the present study ('43-item version' column), 21 were excluded due to the low variability in the responses (ie, ratio from the first to second most populated categories higher than 70/30). Therefore, 22 items were kept for the main analyses ('Reduced version' column). The latent scores resulting from the CFA models computed for both the 43-item and 22-item solutions showed a high correlation $(\mathrm{r}=0.970)$.

\section{Main analyses}

The goodness-of-fit indices of the multigroup SEM models computed for each of the three SWB outcomes are shown in the upper section of online supplemental table S3. All the different models showed good fit (CFI/ TLI $>0.95$ and RMSEA <0.06), and the highest level of invariance tested (ie, equal regression coefficients) was achieved for all SWB outcomes. Therefore, the models with invariant regression coefficients were selected for interpretation.

All item loadings on the respective latent factors were statistically significant $(p<0.001)$. The standardised loadings ranged from 0.523 to 0.753 for physical environmental factors; from 0.539 to 0.819 for social network and support; from 0.340 to 0.731 for functional ability; from 0.446 to 0.835 for evaluative well-being; from 0.732 to 0.817 for PA; and from 0.323 to 0.838 for NA. The visual depictions of the structural models for each of the SWB 


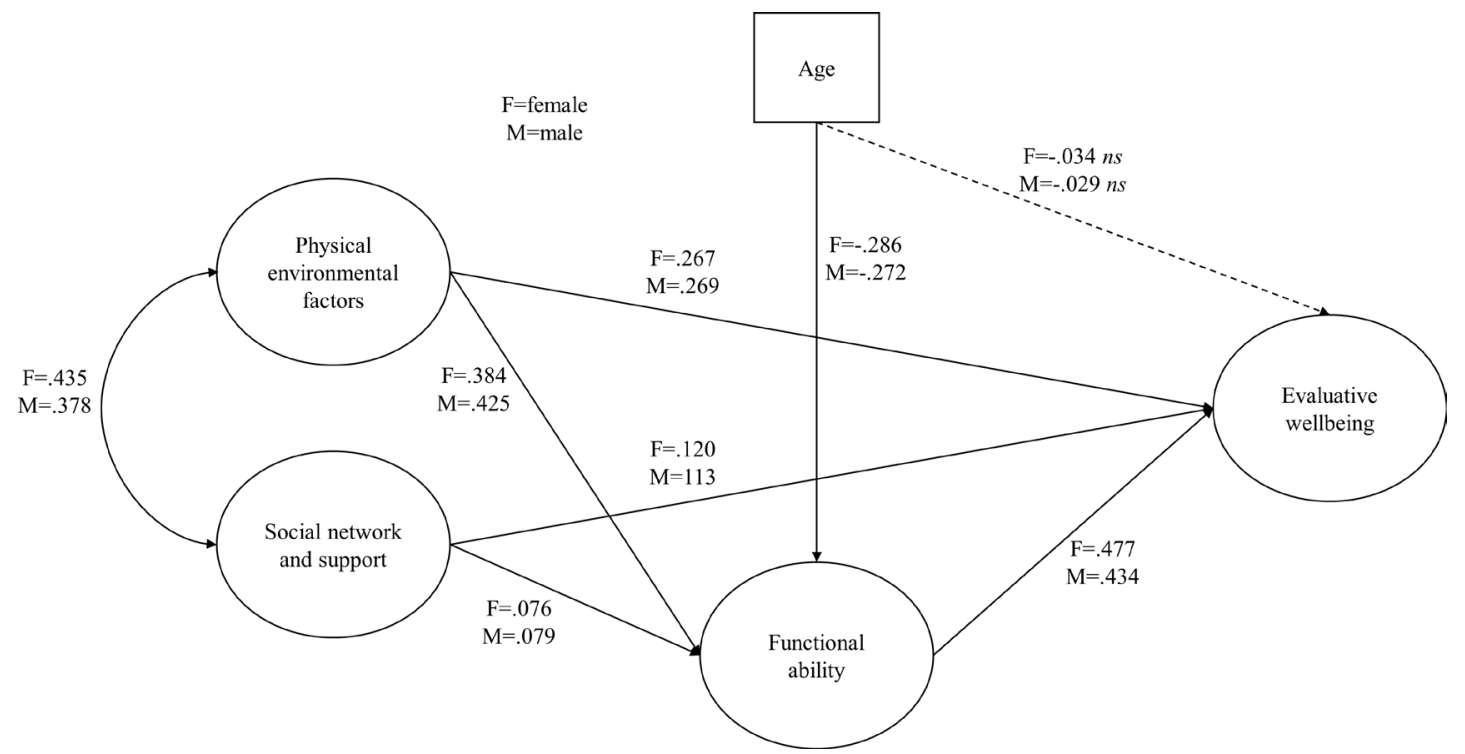

Figure 1 Diagram of the structural equation model performed with evaluative well-being as main outcome. Dashed lines correspond to paths with non-significant (ns) regression coefficients. All coefficients are standardised.

outcomes, including the standardised coefficients for female and male, are shown in figure 1 (evaluative wellbeing), figure $2(\mathrm{PA})$ and figure $3(\mathrm{NA})$. The measurement models of each of the latent variables (including the observed items, loadings, and residuals) are not included in these figures for the sake of clarity. Although regression invariance was achieved, the standardised coefficients are not identical across females and males due to different variances of the observed variables. In all cases, physical environmental factors and social network and support were positively correlated, and were significantly related to higher levels of functional ability. However, the standardised regression coefficients were higher for the physical environmental factors than for social network and support.
The direct effects of functional ability on the three SWB outcomes were, in all cases, statistically significant and positive (ie, higher evaluative well-being and PA, lower NA), with the highest values for NA and the lowest for PA. The direct effects of physical environmental factors on SWB were found to be positive and significant for evaluative well-being and PA, but non-significant for NA; whereas the direct effects of social network and support were positive (ie, higher evaluative well-being and PA, lower NA) and significant in all cases. The standardised indirect effects of the two environmental factors' dimensions on SWB via functional ability, along with the standardised total effects (ie, the sum of the standardised indirect and direct effects), are shown in the upper-left section of table 2. These indirect effects were found to be

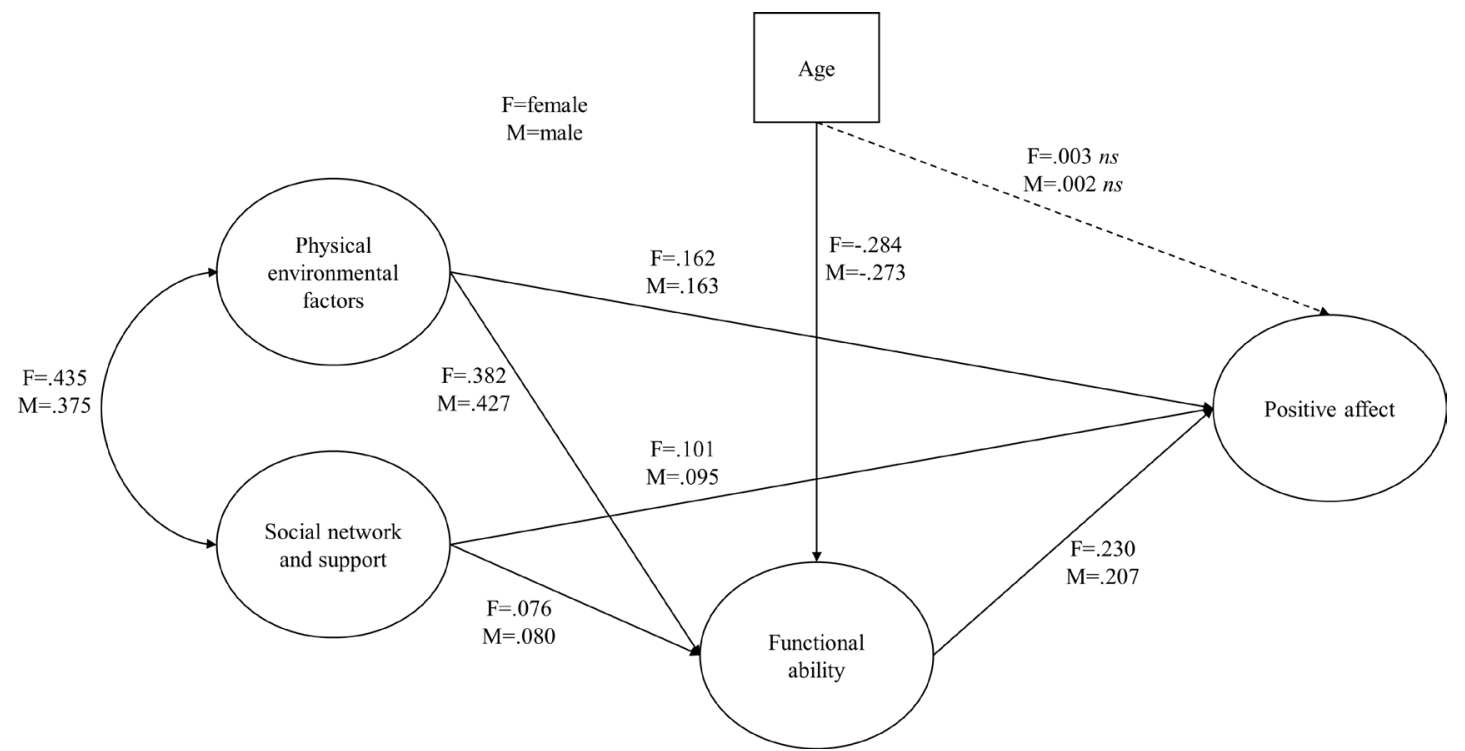

Figure 2 Diagram of the structural equation model performed with positive affect as main outcome. Dashed lines correspond to paths with non-significant (ns) regression coefficients. All coefficients are standardised. 


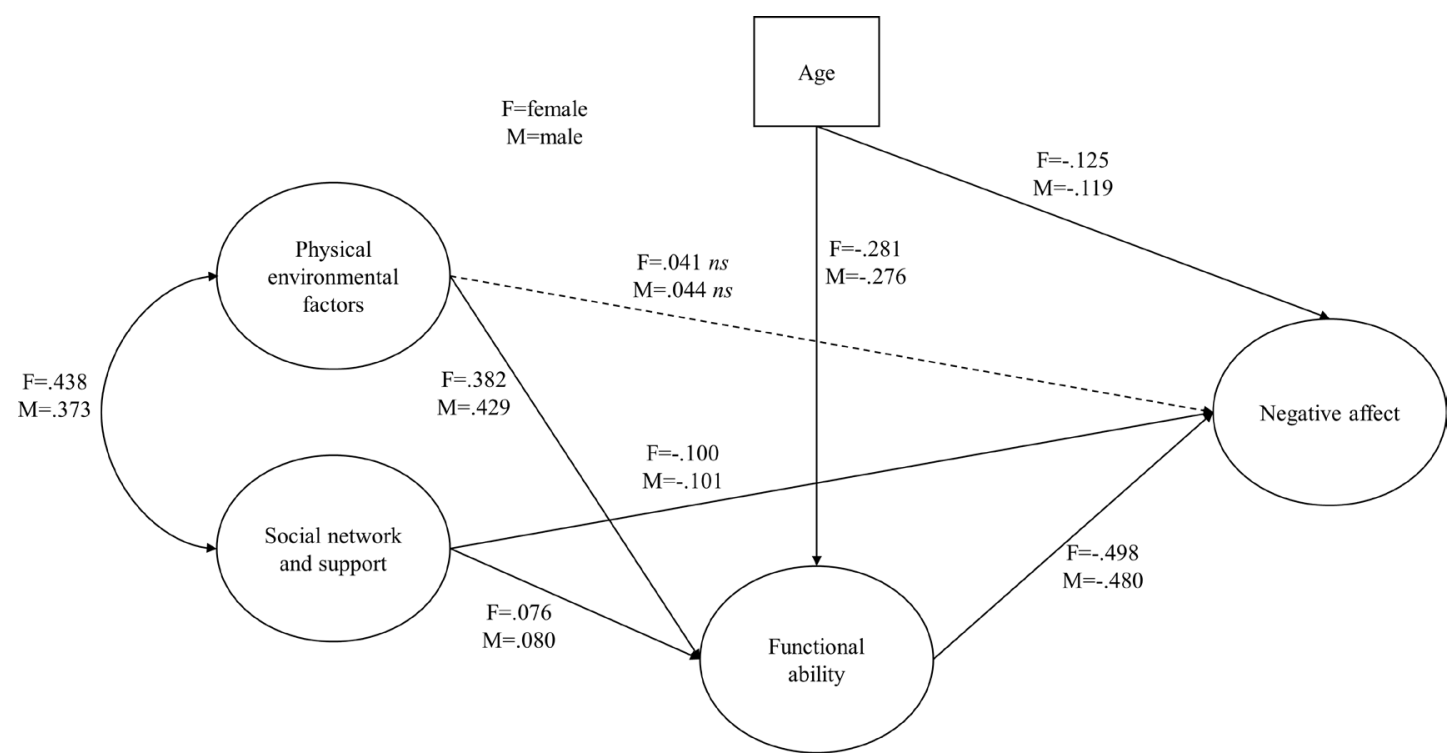

Figure 3 Diagram of the structural equation model performed with negative affect as main outcome. Dashed lines correspond to paths with non-significant (ns) regression coefficients. All coefficients are standardised.

statistically significant in all cases and were larger for physical environmental factors than for social network and support, where most of the effect was direct. The upper section of online supplemental table S4 shows the unstandardised direct, indirect and total effects, along with the 95\% CIs for the direct effects and the bootstrapped $95 \%$ CIs for the indirect and total effects of the main analyses. The coefficients of determination $\left(\mathrm{R}^{2}\right)$ for the models are included in the upper-right section of table 2. Overall, the models explained around $25.6 \%-29.2 \%$ of the variance of the functional ability latent factor, $43.4 \%-48.5 \%$ of the evaluative well-being factor, $13.0 \%-14.5 \%$ of the PA factor and $22.6 \%-24.4 \%$ of the NA factor.

\section{Sensitivity analyses}

The goodness-of-fit indices of the SEM models performed with the brief version of the MDS (see table 1, 'MDS brief version' column) are shown in the lower section of online supplemental table S3. The sample analysed in these models was smaller than that of the main analyses $(n=1621)$, since some included items (ie, work and schooling related) had a high missingness. As in the case of the main analyses, the regression invariance was also achieved for the three SWB outcomes, and the fit of the regression invariant models was good $(\mathrm{CFI} / \mathrm{TLI}>0.95$, RMSEA <0.06) and all item loadings in the models were significant $(p<0.001)$. The visual depictions of the regression invariant SEM models with the standardised coefficients are included in online supplemental figure S1 (evaluative well-being), online supplemental figure S2 (PA) and online supplemental figure S3 (NA) in the supplementary material, again excluding the measurement model (indicators, loadings and residuals) for a clearer depiction. The main difference between the main analyses and the sensitivity analyses using the brief version of the MDS was that, in the latter, the indirect effect of social network and support on all SWB outcomes, along

Table 2 Standardised indirect and total effects of the environmental factors and percentage of variance explained of outcomes in main and sensitivity analyses

\begin{tabular}{|c|c|c|c|c|c|c|c|c|c|c|c|c|c|}
\hline & & \multicolumn{4}{|c|}{ Physical environmental factors } & \multicolumn{4}{|c|}{ Social network and support } & \multicolumn{4}{|l|}{$\mathbf{R}^{2}$} \\
\hline & & \multicolumn{2}{|c|}{ Indirect effect } & \multicolumn{2}{|c|}{ Total effect } & \multicolumn{2}{|c|}{ Indirect effect } & \multicolumn{2}{|c|}{ Total effect } & \multicolumn{2}{|c|}{$\begin{array}{l}\text { Functional } \\
\text { ability }\end{array}$} & \multicolumn{2}{|c|}{ SWB outcome } \\
\hline & & Estimate & SE & Estimate & SE & Estimate & SE & Estimate & SE & Female & Male & Female & Male \\
\hline \multirow{3}{*}{$\begin{array}{l}\text { Main } \\
\text { analyses }\end{array}$} & EvWB & $0.183^{\star \star \star}$ & 0.012 & $0.450^{\star \star \star}$ & 0.021 & $0.036^{\star *}$ & 0.009 & $0.156^{\star \star \star}$ & 0.021 & 0.260 & 0.286 & 0.485 & 0.434 \\
\hline & PA & $0.088^{\star \star \star}$ & 0.012 & $0.250^{\star \star \star}$ & 0.028 & $0.018^{\star *}$ & 0.007 & $0.119^{\star \star \star}$ & 0.033 & 0.258 & 0.289 & 0.145 & 0.130 \\
\hline & NA & $-0.190^{\star * *}$ & 0.009 & $-0.149^{\star \star \star}$ & 0.011 & $-0.038^{\star \star}$ & 0.006 & $-0.138^{\star \star *}$ & 0.014 & 0.256 & 0.292 & 0.244 & 0.226 \\
\hline \multirow{2}{*}{$\begin{array}{l}\text { Sensitivity } \\
\text { analyses }\end{array}$} & PA & $0.102^{\star * \star}$ & 0.016 & $0.243^{\star \star \star}$ & 0.034 & 0.015 & 0.010 & $0.122^{* *}$ & 0.042 & 0.232 & 0.287 & 0.161 & 0.126 \\
\hline & NA & $-0.157^{\star \star \star}$ & 0.009 & $-0.121^{\star \star \star}$ & 0.014 & -0.023 & 0.007 & $-0.093^{\star \star}$ & 0.016 & 0.229 & 0.291 & 0.170 & 0.148 \\
\hline
\end{tabular}

Effects correspond to the standardised solution.

${ }^{* *} p<0.01 ;{ }^{* * *} p<0.001$.

EvWB, evaluative well-being; NA, negative affect; PA, positive affect; $R^{2}$, coefficient of determination; SE, bootstrapped SE. 
with its direct effect on functional ability, were found to be statistically non-significant (table 2 , lower left section). The lower section of online supplemental table S4 shows the unstandardised direct, indirect and total effects, along with the $95 \%$ CIs for the direct effects and the bootstrapped $95 \%$ CIs for the indirect and total effects of the sensitivity analyses. The remaining effects were found to be similar to those in the main analyses, although the coefficients of determination (table 2, lower right section) were smaller in these sensitivity analyses.

The results from the models carried out with the imputed data are available in online supplemental table S5 (fit indices, standardised indirect and total effects and coefficients of determination), online supplemental figure S4 (evaluative well-being), online supplemental figure S5 (PA) and online supplemental figure S6 (NA) in the supplementary material. These models included all participants with valid information in any of the variables (46 participants were excluded), thus rendering a sample size of $n=2779$. The results went in the same direction as those from the main analyses: the indirect effects through both physical environmental factors and social network and support were statistically significant in all cases, larger for physical environmental factors than for social network and support, and very similar in their size as those found in the main analyses.

\section{DISCUSSION}

The models presented in this paper showed empirical support for the concept of functional ability and for the WHO framework on healthy ageing. We found that substantial proportions of the effects of environmental factors and social networks on the three SWB components were taking place through enhanced levels of functional ability. We also found that the models were equivalent for both men and women, confirming the equivalent effect, both direct and indirect, of environmental factors. Whether this is a finding that is specific to the country the study was carried out, or whether it is more generalisable beyond the Philippines, should be investigated in further research.

In this particular sample (ie, Philippine older adults), our reduced version of the MDS captured in a roughly equivalent manner the functional ability of participants than a more extensive measure including almost twice the items. Moreover, the models were similar in both analyses using our reduced version of the MDS items and the official brief MDS version, with the exception that functional ability did not account for any portion of the effect of social network and support on any of the SWB outcomes when using the brief MDS version. This could reflect the relatively lower presence of social-oriented items in the brief version of the MDS, thus leading to a reduction in the impact of the social network and support on that operationalisation of functional ability. This is likely to be possible as items related to "difficulty in engaging in local or national politics and in civil society organisations, such as Rotary Club, Red Cross', or in 'providing care and support for others' are excluded from the brief version of the MDS. Future studies may put to test whether the brief MDS appropriately captures functional ability among older adults, and whether our empirically driven abbreviated version is adequate for samples with other characteristics such as different age ranges or geographical contexts. Nevertheless, social support appeared to have smaller impact on SWB and functional ability compared with physical environmental factors, in both the main analysis and the sensitivity analysis. This may be a reflection of the location of the study, the Philippines, where environmental factors in older age may be more influential than social support, or that there is a more culturally embedded social network, leading to a smaller relative impact. It is also plausible that a good portion of the effect of physical environmental factors on SWB takes place through functional ability, as it is less likely that the facilities and infrastructural characteristics per se provide SWB, whereas the social support can more easily have a direct impact on how people feel and value their lives. The impact of physical and social environmental factors on healthy ageing is consistent with other studies that were carried out in older age. A review of the role of environment for healthy ageing provided several examples of the associations between both social and physical environmental factors and healthy ageing. ${ }^{14}$ Most of the evidence reviewed corresponded to the latter, highlighting the importance of supportive, barrier-free environments but most particularly at the physical level (eg, street conditions, noise, availability and quality of the transportation). More recently, a study by Gobbens and van Assen reported strong associations between multiple environmental factors and quality of life in a sample of older Dutch individuals. ${ }^{28}$ The role of social contact and participation in community activities in healthy ageing has also been highlighted by multiple studies. In a study of positive ageing trajectories, it was shown that participants with higher levels of social support were more likely to be in a 'high health trajectory' compared with a low 'health trajectory' (OR: $1.13,95 \%$ CI: 1.08 to 1.19$){ }^{29}$ The impact of participating in community activities has also been shown in many other longitudinal studies, suggesting that a higher participation in social activities is positively associated with healthy ageing. ${ }^{30-33}$ Although these epidemiological associations are very important, emerging evidence has also started appearing on the role that interventions may have in changing physical and social environmental factors. ${ }^{34}$ For example, a smallscale intervention on walking behaviour among older people living in the Netherlands showed that both the physical intervention and the social interventions had an impact on the total time spent walking for all conditions. Although this study focused on walking and did not report on any other outcomes, it showed that physical and social environmental interventions can be implemented in older age. ${ }^{35}$ 


\section{Strengths and limitations}

We used a multidimensional approach to SWB, acknowledging that different components can display different relationships with other constructs. ${ }^{36}$ Sensitivity analyses were used to analyse the impact of different operationalisations of the functional ability construct in those relationships. The use of a multigroup approach acknowledging the potential moderating effect of gender in the network of relationships of the healthy ageing concept is also worth nothing, as the WHO ageing report clearly acknowledges that gender can interact with the 'exposures, opportunities and barriers we face, as well as our access to resources', ${ }^{1}$ and age and gender differences in functional ability have also been reported among older adults. $^{5}$

Although this is to our knowledge one of the first studies to provide empirical support to the WHO framework on healthy ageing using data from a low- and middle-income country, there are still several limitations that need to be taken into consideration. The analysis used data from the MDS. Although this dataset has very comprehensive measures of functional ability, it lacks objective intrinsic capacity measures. This, paired with the high statistical correlation between functional ability and the intrinsic capacity operationalisation used in the MDS dataset, meant that we were unable to include the latter in our statistical models. The data we had access to were also only cross-sectional, which meant that our analysis could not take any temporality into account and that reverse causation is still a potential problem. Moreover, the crosssectional design prevented us from modelling dynamics such as the individual shaping their environments. Therefore, future analyses should test these relationships using a longitudinal approach. It is also important to highlight that the environmental factors contained in the MDS that were used for the analyses were not specifically tailored to ageing populations, and that other indicators that may be more applicable to older adults should be tested in future research. Moreover, although the acknowledgement of the social environmental aspects goes further than much of the available literature which often accounts exclusively for the physical environmental characteristics, ${ }^{14}$ it is far from exhaustingly covering all the environmental characteristics that may play a role in healthy ageing. Therefore, future works may account for the dynamic nature of the environmental factors considered in this study, along with other relevant aspects such as the social and political systems and policies, that could not be accounted for in this study due to the nature of the study design (crosssectional) and data available (individual data).

\section{CONCLUSIONS AND FUTURE STEPS}

Our findings provide support to the healthy ageing framework of the WHO and specifically to the concept of functional ability. They also provide information on multiple domains of functional ability, whereas comparative information available across 37 countries is currently limited to an incomplete assessment of one domain. ${ }^{5}$ In this paper, we have shown that environmental factors play an important role in SWB, and that those associations are both direct and indirect, partly explained by functional ability. This provides more evidence for the robustness of the functional ability concept, and for the importance that physical and social environmental factors have in shaping how we can age healthily, achieving well-being in older age. This is just the beginning of the puzzle, and more empirical research over time and in diverse settings is needed. This should include the analysis of datasets that have strong information on functional ability, together with objective measures of intrinsic capacity, so that this latter concept could also be fitted in the model. There is also the need for future surveys to include strong and reliable indicators of both functional ability and of intrinsic capacity that were developed for the purpose of measuring those concepts, rather than informing specific research questions of interest to the investigators, as commonly seen across studies. ${ }^{6}$ In this regard, the WHO has described recently an agenda to measure all areas of functional ability, intrinsic capacity and environmental factors. ${ }^{5}$ It is also important that future studies add a longitudinal component to these analyses, as currently the assessment of functional ability has had mainly a cross-sectional focus. Elucidating the life-course impact of environmental and social support factors, while taking into account gender, will also help us in developing policies aimed at improving our functional ability capacities at different stages of life to better prepare us for older age.

\section{Author affiliations \\ ${ }^{1}$ Health Service and Population Research, King's College London, London, UK ${ }^{2}$ Centre for Longitudinal Studies, UCL Social Research Institute, University College London, London, UK \\ ${ }^{3}$ ESRC Centre for Society and Mental Health, King's College London, London, UK ${ }^{4}$ Ageing and Health Unit, Maternal, Newborn, Child \& Adolescent Health \& Ageing Department, World Health Organization, Geneva, Switzerland \\ ${ }^{5}$ Demographic Change and Healthy Ageing Unit, Social Determinants of Health Department, World Health Organization, Geneve, Switzerland \\ ${ }^{6}$ Sensory Functions, Disability and Rehabilitation Unit, Department of Noncommunicable Diseases, World Health Organization, Geneve, Switzerland}

Twitter Dario Moreno-Agostino @dmorenoagostino and Matthew Prina @am_prina Contributors DMA, MP, KK and AC conceived the work. DMA performed the analyses. KCC supervised the analyses. DMA and MP wrote the first draft. KCC, ATJ, $\mathrm{RS}, \mathrm{AO}, \mathrm{KK}$ and $\mathrm{AC}$ provided critical feedback and contributed to the interpretation of the findings and writing of the final manuscript. All authors read and approved the final manuscript.

Funding This work was supported by the ESRC Centre for Society and Mental Health at King's College London, grant number ES/S012567/1. The views expressed are those of the author(s) and not necessarily those of the ESRC or King's College London.

Competing interests None declared.

Patient consent for publication Not applicable.

Ethics approval Informed consent and ethical approval were obtained by the local government. The MDS survey underwent the Statistical Survey Review and Clearance System (SSRCS) by the Philippine Statistics Authority, with approval numbers PSA-1635-01 and PSA-1635-02.

Provenance and peer review Not commissioned; externally peer reviewed. 
Data availability statement The data analysed is available upon request from the Philippine Statistics Authority (https://psa.gov.ph/content/data-request-form).

Supplemental material This content has been supplied by the author(s). It has not been vetted by BMJ Publishing Group Limited (BMJ) and may not have been peer-reviewed. Any opinions or recommendations discussed are solely those of the author(s) and are not endorsed by BMJ. BMJ disclaims all liability and responsibility arising from any reliance placed on the content. Where the content includes any translated material, BMJ does not warrant the accuracy and reliability of the translations (including but not limited to local regulations, clinical guidelines, terminology, drug names and drug dosages), and is not responsible for any error and/or omissions arising from translation and adaptation or otherwise.

Open access This is an open access article distributed in accordance with the Creative Commons Attribution Non Commercial (CC BY-NC 4.0) license, which permits others to distribute, remix, adapt, build upon this work non-commercially, and license their derivative works on different terms, provided the original work is properly cited, appropriate credit is given, any changes made indicated, and the use is non-commercial. See: http://creativecommons.org/licenses/by-nc/4.0/.

\section{ORCID iDs}

Dario Moreno-Agostino http://orcid.org/0000-0003-0459-657X

Kaloyan Kamenov http://orcid.org/0000-0002-4355-6186

\section{REFERENCES}

1 World Health Organization. World report on ageing and health; 2015.

2 Belloni G, Cesari M. Frailty and intrinsic capacity: two distinct but related constructs. Front Med 2019;6:133.

3 World Health Organization. Global strategy and action plan on ageing and health; 2017.

4 Cesari M, Araujo de Carvalho I, Amuthavalli Thiyagarajan J, et al. Evidence for the domains supporting the construct of intrinsic capacity. J Gerontol A Biol Sci Med Sci 2018;73:1653-60.

5 World Health Organization. Decade of healthy ageing: baseline report. Geneva: World Health Organization, 2020.

6 Beard JR, Jotheeswaran AT, Cesari M, et al. The structure and predictive value of intrinsic capacity in a longitudinal study of ageing. BMJ Open 2019;9:e026119.

7 Yu R, Amuthavalli Thiyagarajan J, Leung J, et al. Validation of the construct of intrinsic capacity in a longitudinal Chinese cohort. J Nutr Health Aging 2021;25:808-15.

$8 \mathrm{Ma} \mathrm{L}$, Chhetri JK, Zhang L, et al. Cross-Sectional study examining the status of intrinsic capacity decline in community-dwelling older adults in China: prevalence, associated factors and implications for clinical care. BMJ Open 2021;11:e043062.

9 Gutiérrez-Robledo LM, García-Chanes RE, González-Bautista E, et al. Validation of two intrinsic capacity scales and its relationship with frailty and other outcomes in Mexican community-dwelling older adults. J Nutr Health Aging 2021;25:33-40.

10 World Health Organization. Strengthening the collection of data on disability, 2021. Available: https://www.who.int/activities/collectionof-data-on-disability [Accessed 21 July 2021].

11 Philippine Statistics Authority. 2016 national disability prevalence survey, 2019. Available: https://psa.gov.ph/sites/default/files/2016\% 20NDPS.pdf [Accessed 21 July 2021].

12 International Household Survey Network. Model functioning survey, NDPS/MFS form 2. individual questionnaire, 2016. Available: https:// catalog.ihsn.org/index.php/catalog/7223/download/86174 [Accessed 21 Jul 2021].

13 World Health Organization. International classification of functioning, disability and health: ICF. Geneva World Health Organization; 2001.

14 Clarke P, Nieuwenhuijsen ER. Environments for healthy ageing: a critical review. Maturitas 2009;64:14-19.
15 Leonardi M, Chatterji S, Koskinen S, et al. Determinants of health and disability in ageing population: the COURAGE in Europe project (Collaborative research on ageing in Europe). Clin Psychol Psychother 2014;21:193-8.

16 Dolan P, Kudrna L, Stone A. The measure matters: an investigation of evaluative and experience-based measures of wellbeing in time use data. Soc Indic Res 2017;134:57-73.

17 Dolan P, Metcalfe R. Measuring subjective wellbeing: recommendations on measures for use by National governments. $J$ Soc Policy 2012;41:409-27.

18 Diener E. Guidelines for national indicators of subjective well-being and III-Being. J Happiness Stud 2006;7:397-404.

19 Gregorich SE. Do self-report instruments allow meaningful comparisons across diverse population groups? testing measurement invariance using the confirmatory factor analysis framework. Med Care 2006;44:S78.

20 Bentler PM. Cutoff criteria for fit indexes in covariance structure analysis: conventional criteria versus new alternatives. Structural Equation Modeling: A Multidisciplinary Journal 1999;6:1-55.

21 Chen FF. Sensitivity of Goodness of fit indexes to lack of measurement invariance. Structural Equation Modeling: A Multidisciplinary Journal 2007;14:464-504.

22 Cheung GW, Rensvold RB. Evaluating goodness-of-fit indexes for testing measurement invariance. Structural Equation Modeling: A Multidisciplinary Journal 2002;9:233-55.

23 World Health Organization. Brief model disability survey (brief MDS). Implementation guide, 2017. Available: https://www.who.int/ disabilities/data/Implementation-guide-Brief-MDS.pdf [Accessed 21 Jul 2021].

24 Sterne JAC, White IR, Carlin JB, et al. Multiple imputation for missing data in epidemiological and clinical research: potential and pitfalls. BMJ 2009;338:b2393.

25 The R Foundation. R: A Language and Environment for Statistical Computing [program]. 3.6.3 version. Vienna, Austria R Foundation for Statistical Computing; 2020.

26 Rosseel Y. lavaan : An $R$ Package for Structural Equation Modeling. J Stat Softw 2012;48:1-36.

27 Buuren Svan, Groothuis-Oudshoorn K. mice : Multivariate Imputation by Chained Equations in R. J Stat Softw 2011;45:1-67.

28 Gobbens RJJ, van Assen MALM. Associations of environmental factors with quality of life in older adults. Gerontologist 2018;58:101-10.

29 Zaslavsky O, Cochrane BB, Woods NF, et al. Trajectories of positive aging: observations from the women's health Initiative study. Int Psychogeriatr 2014;26:1351-62.

30 Pruchno RA, Wilson-Genderson M. A longitudinal examination of the effects of early influences and midlife characteristics on successful aging. J Gerontol B Psychol Sci Soc Sci 2015;70:850-9.

$31 \mathrm{Hsu} \mathrm{H}-\mathrm{C}$. Trajectories of multimorbidity and impacts on successful aging. Exp Gerontol 2015;66:32-8.

32 Liu L-F, Su P-F. What factors influence healthy aging? A personcentered approach among older adults in Taiwan. Geriatr Gerontol Int 2017;17:697-707.

33 Hodge AM, English DR, Giles GG, et al. Social connectedness and predictors of successful ageing. Maturitas 2013;75:361-6.

34 Giles-Corti B, Donovan RJ. The relative influence of individual, social and physical environment determinants of physical activity. Soc Sci Med 2002;54:1793-812.

35 Prins RG, Kamphuis CBM, Van Lenthe FJ. The effects of small-scale physical and social environmental interventions on walking behaviour among Dutch older adults living in deprived neighbourhoods: results from the quasi-experimental NEW.ROADS study. Int J Behav Nutr Phys Act 2019;16:133.

36 Moreno-Agostino D, de la Torre-Luque A, de la Fuente J. Determinants of subjective wellbeing trajectories in older adults: a growth mixture modeling approach. Journal of Happiness Studies2020. 